This item was submitted to Loughborough's Research Repository by the author.

Items in Figshare are protected by copyright, with all rights reserved, unless otherwise indicated.

\title{
High-throughput single-shot hyperspectral interferometer for areal profilometry based on microlens array integral field unit
}

PLEASE CITE THE PUBLISHED VERSION

https://doi.org/10.1117/12.2272003

PUBLISHER

(c) SPIE

VERSION

AM (Accepted Manuscript)

LICENCE

CC BY-NC-ND 4.0

\section{REPOSITORY RECORD}

Ruiz, Pablo D., and Jonathan M. Huntley. 2019. "High-throughput Single-shot Hyperspectral Interferometer for Areal Profilometry Based on Microlens Array Integral Field Unit”. figshare. https://hdl.handle.net/2134/24829. 


\title{
High-throughput single-shot hyperspectral interferometer for areal profilometry based on microlens array integral field unit
}

\author{
Pablo D. Ruiz* and Jonathan M. Huntley \\ Wolfson School of Mechanical, Electrical and Manufacturing Engineering, \\ Loughborough University, Loughborough, UK, LE11 3TU
}

\begin{abstract}
A single-shot technique to measure areal profiles on optically smooth and rough surfaces and for applications in noncooperative environments is presented. It is based on hyperspectral interferometry (HSI), a technique in which the output of a white-light interferometer provides the input to a hyperspectral imaging system. Previous HSI implementations suffered from inefficient utilisation of the available pixels which limited the number of measured coordinates and/or unambiguous depth range. In this paper a $>20$-fold increase in pixel utilization is achieved through the use of a 2 -D microlens array as proposed for integral field units in astronomy applications. This leads to a $35 \times 35$ channel system with an unambiguous depth range of $0.88 \mathrm{~mm}$.
\end{abstract}

Keywords: height measurements, metrology, single-shot, hyperspectral interferometry, microlens array, integral field unit.

\section{INTRODUCTION}

Scanning white light interferometry (SWLI) has become an established technique to measure absolute distance and surface profile of components with height steps and steep height gradients. The use of a broadband source eliminates the need for phase unwrapping and increases the unambiguous measurement range with respect to systems that use monochromatic light. In SWLI, the object or a Mirau objective is scanned axially while keeping the reference beam stationary ${ }^{1-3}$. The surface height with respect to a datum is then found by detecting the point of maximum fringe modulation on a pixelwise basis. In a closely related technique, known as wavelength (or frequency) scanning interferometry (WSI or FSI), the optical path difference between both arms in the interferometer remains fixed while the wavelength is scanned over time ${ }^{4-5}$. The scanning nature of these approaches imposes stringent environmental stability requirements during the acquisition of typically hundreds of interferograms. To overcome this limitation, a 'single-shot' method has been proposed in which all the spatial and spectral information is recorded simultaneously in a single exposure $^{6-7}$. Known as hyperspectral interferometry (HSI), the output of a white-light interferometer provides the input to a hyperspectral imaging system to spatially separate, onto a photodetector array, a set of narrowband interferograms from a single white-light interferogram. Unfortunately, existing implementations based on an etalon filter and a diffraction grating use only $\sim 2 \%$ of the available pixels, which limits the number of measured coordinates to $\sim 200$ pixels with an unambiguous depth range of $\sim 0.3 \mathrm{~mm}$.

In this paper, in order to spatially separate the spectral information onto the photodetector array we use a microlens array that leads to a pixel usage of over $40 \%$, i.e. a $20 \times$ improvement on previous HSI setups. The concept has been adapted from integral field units used in astronomy, where pixel usage and throughput are optimized ${ }^{8}$. Using a Linnik type interferometer, the object is imaged onto a microlens array, where it interferes with the reference beam. Behind the microlens array white light spots are produced, which are then dispersed by a grating and imaged as an array of linear spectra onto a large area photodetector array. For each microlens, the optical path between its corresponding point on the object and the reference beam determines a modulation frequency along the wavelength axis of its spectrum. Fourier transformation along each of the spectra in the wavenumber domain leads to a 2-D map of surface height.

* p.d.ruiz@lboro.ac.uk, phone +44 1509227660 


\section{SYSTEM DESCRIPTION}

\subsection{Optical Setup}

The system consists of a Linnik-type imaging interferometer and a hyper-spectral imager - see Fig. 1. A superluminescent light emitting diode, SLD, is used as the light source (Superlum Diodes Ltd, $840 \mathrm{HP} 1 ; 15 \mathrm{~mW}$ output power; centre wavelength $\lambda_{\mathrm{c}}=840 \mathrm{~nm}$; bandwidth $\Delta \lambda=50 \mathrm{~nm}$ ). Two different configurations are used to launch light into the interferometer, as shown schematically in the top left of Fig. 1 and described below.

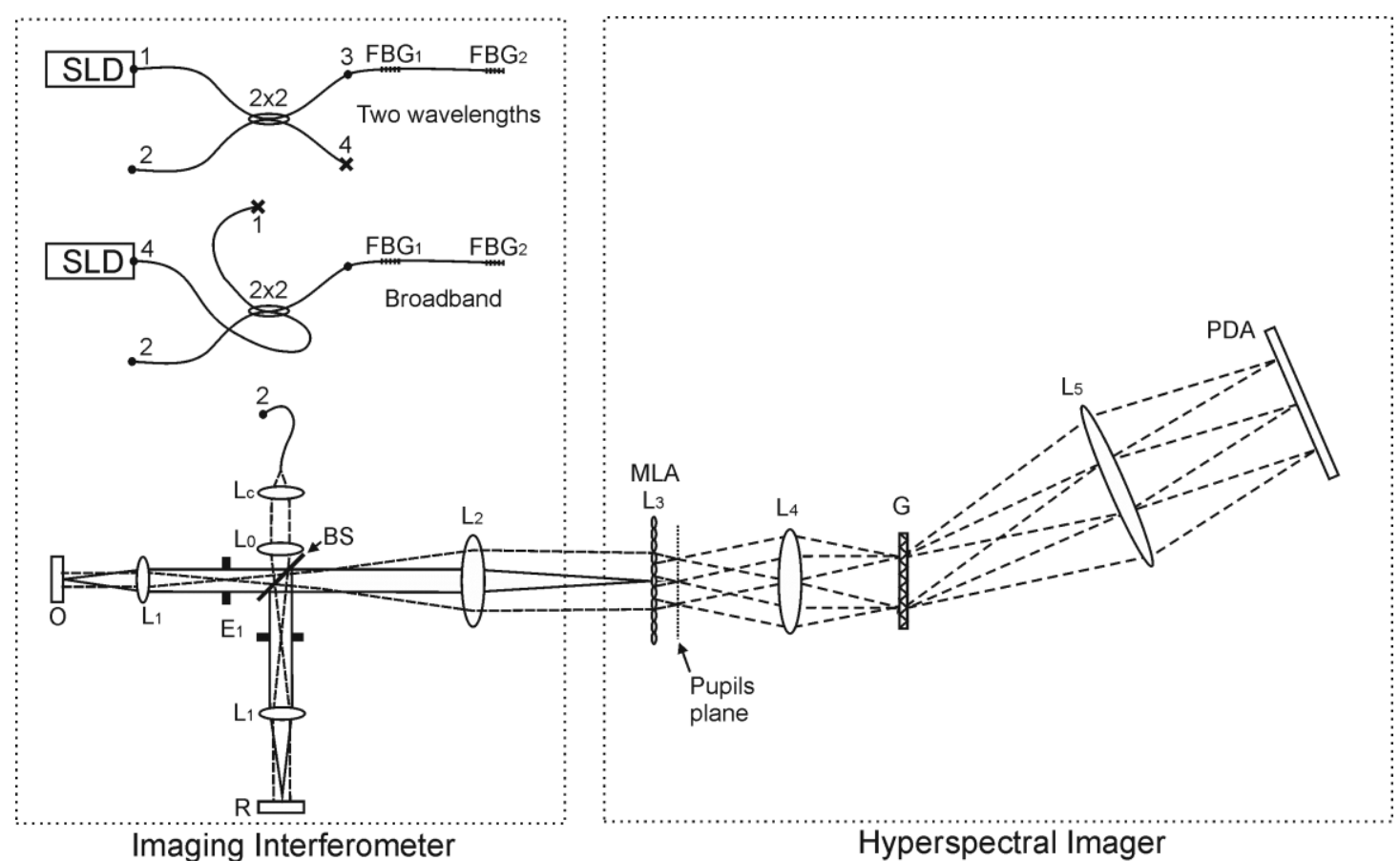

Figure 1. Optical setup of the Hyperspectral Interferometry system based on a microlens array, showing: SLD: Superluminescent light emitting diode; FBG1, FBG2: Fibre Bragg gratings; Lc: collimator; BS: beam splitter; O: object; R: reference mirror; $\mathrm{L}_{0}, \mathrm{~L}_{1}, \mathrm{~L}_{2}, \mathrm{~L}_{4}$ and $\mathrm{L}_{5}$ : NIR achromatic lenses; $\mathrm{E}_{1}$ : aperture stop; MLA: microlens array; $\mathrm{L}_{3}$ : lenslet; $\mathrm{G}$ : diffraction grating and PDA: photodetector array. At the top of the imaging interferometer box, the 'two wavelengths' and 'broadband' illumination configurations are shown.

1) 'Two wavelengths' illumination. In this configuration, the output of the SLD is connected to fibre (1) of a $2 \times 2$, 90:10 fibre coupler. Fibre (3) of the coupler is connected to a single mode fibre with two fibre Bragg gratings $\mathrm{FBG}_{1}$ and $\mathrm{FBG}_{2}$ which act as spectrally selective mirrors for wavelengths $\lambda_{1}=820 \mathrm{~nm}$ and $\lambda_{2}=853 \mathrm{~nm}$ with a narrow spectral bandwidth $(<0.01 \mathrm{~nm})$. Light reflected at $\mathrm{FBG}_{1}$ and $\mathrm{FBG}_{2}$ is delivered to the interferometer via fibre (2) of the fibre coupler. This configuration is used to calibrate the wavelength axis in the spectral images.

2) 'Broadband' illumination. In this configuration, fibre (4) of the fibre coupler is connected to the output of the SLD source, bypassing the fibre Bragg gratings and delivering broadband light directly to the interferometer via fibre (2) of the fibre coupler, as in the two wavelengths configuration.

At the interferometer's input, collimator $\mathrm{L}_{c}$, lens $\mathrm{L}_{0}$ and beam splitter BS produce a pair of point sources at a distance $f_{0}$ from $\mathrm{L}_{0}$, one on each arm of the interferometer. The point sources are at the back focal planes of lenses $\mathrm{L}_{1}$, which collimate the beam onto the reference mirror and the object. Doubly telecentric systems formed by lenses $\mathrm{L}_{1}$ (at both reference and object arms) and $\mathrm{L}_{2}$ image the reference mirror and the object at the back focal plane of $\mathrm{L}_{2}$. At that plane, 
an image of the object is recombined with a collimated reference beam so as to form a broadband interferogram. The output of the interferometer, i.e. the image of the object recombined with the reference beam, acts as the input for a hyper-spectral imager. At the image plane, a microlens array, MLA, spatially samples the image of the object (Suss MicroOptics, fused silica refractive index $n_{r}=1.4525$ at $840 \mathrm{~nm}$, lenslet diameter $100 \mu \mathrm{m}$, arranged on a square grid of pitch $100 \mu \mathrm{m}$, regions between lenses chrome-coated, radius of curvature ROC $=60 \mu \mathrm{m}$, effective focal length $f=$ $\mathrm{ROC} /\left(n_{r}-1\right)=130 \mu \mathrm{m}$, numerical aperture NA $=0.38$, array size $\left.=5 \times 5 \times 1.2 \mathrm{~mm}^{3}\right)$. A given lenslet labelled by indices $(m$, $n$ ) collects light from a small region on the sample surface, centred on the point with coordinates $\left(x_{m}, y_{n}\right)$, where $m=$ $0,1,2, \ldots, N_{x}-1, n=0,1,2, \ldots, N_{y}-1$, and $N_{x}, N_{y}$ are respectively the number of lenslets along the $x$ and $y$ axes.

At the back focal plane of each lenslet (the pupil plane) a diffraction pattern is formed. When a flat wavefront is incident on the lenslet, the diffraction pattern corresponds to the point spread function (PSF) of the lenslet. The resulting array of pupils is imaged with a doubly telecentric system formed by lenses $\mathrm{L}_{4}$ and $\mathrm{L}_{5}$ onto a large format photodetector array, PDA (Apogee U16M: 4096×4096 pixels, pixel size $9 \mu \mathrm{m}$, array size $36.86 \mathrm{~mm}$, array diagonal $52.13 \mathrm{~mm}$ ). At the pupil plane of this telecentric system a transmission, near-infrared diffraction grating $\mathrm{G}$ (300 lines $\mathrm{mm}^{-1}$ ) diffracts the collimated beams produced by $\mathrm{L}_{4}$ from each lenslet PSF. The first diffracted order is collected by lens $\mathrm{L}_{5}$ so that the spots at the back focal plane of the MLA become lines in the plane of the PDA. In our MLA, the spots diameter at the pupils plane was $\sim 10 \mu \mathrm{m}$ as implied from their image on the PDA, most likely due to spherical and chromatic aberrations of the microlenses due to their high NA. In order to avoid spectra overlap on the PDA, the MLA is rotated a small angle with respect to the lines on the diffraction grating. The PDA array is aligned so that pixel columns are parallel to the lines of the diffraction grating ${ }^{8}$.

\subsection{Surface height maps}

The procedure to obtain a surface height map from the image of the spectra produced from the MLA pupils consists of the following steps, which are performed for all spectra in the image:

1. Find the coordinates $\left(x_{m, n}^{P D A 1}, y_{m, n}^{P D A 1}\right)$ and $\left(x_{m, n}^{P D A 2}, y_{m, n}^{P D A 2}\right)$ of the points where the reference wavelengths $\lambda_{1}$ and $\lambda_{2}$ fall on the PDA array when the 'Two wavelengths' illumination configuration is used. This is performed for all $(m, n)$ with sub-pixel resolution by fitting a 2-D Gaussian function to the diffraction pattern within each Airy disk.

2. Evaluate the distance, $d(m, n)$, between points $\left(x_{m, n}^{P D A 1}, y_{m, n}^{P D A 1}\right)$ and $\left(x_{m, n}^{P D A 2}, y_{m, n}^{P D A 2}\right)$ and the orientation (angle subtended to the PDA rows) of the line that connects them.

3. Use the distance $d(m, n)$ to scale the spectrum and find the wavelength for all other points along the spectrum. A linear relationship between position and wavelength is assumed, as the residuals relative to the case described by the grating equation do not exceed 0.08 of a pixel between $815 \mathrm{~nm}$ and $865 \mathrm{~nm}$.

4. Evaluate pixel coordinates along the wavelength axis of the spectrum so that it can be re-sampled with equal steps along the wavenumber axis.

5. Interpolate the spectral interferogram, the reference beam spectrum and the object beam spectrum at the pixel coordinates found in step (4).

6. Remove the background intensity, or dc term, by subtracting the reference and object spectra from the spectral interferogram ${ }^{6}$.

7. Multiply the re-sampled, dc-free 1-D intensity signal obtained in step (6) by a Hanning window to reduce phase noise due to crosstalk and then evaluate its Fourier transform.

8. Find the frequency at the maximum of the magnitude of the Fourier transform, which localizes the surface relative to a datum defined by the reference mirror in Fig. 1. This is done with sub-pixel resolution using the continuous Fourier transform ${ }^{9}$.

9. Convert the peak frequency into optical path difference, $z$, using the relationship $z=2 \pi f_{k} / \Delta k$, where $f_{k}$ is the frequency in units of cycles across the spectral bandwidth used, $\Delta k$. 
10. Evaluate the surface height as $h=h_{0}-z / 2$, where $h_{0}$ is the known distance from the sample datum surface to the plane of zero optical path difference.

\section{RESULTS}

Figure 2(a) shows a horizontal profile along one spectrum on the PDA corresponding to one lenslet on the MLA. The spectral range (expressed in wavenumber in the figure) corresponds to $810 \mathrm{~nm}$ to $835 \mathrm{~nm}$. This is a spectral interferogram resulting from Step 7 of section 2.2 in which spatial frequency encodes optical path difference between the reference mirror and the object at the point imaged by the corresponding lenslet in the microlens array. The modulation drops to values close to zero outside the range $k=7.54-7.74 \times 10^{6} \mathrm{~m}^{-1}$, corresponding to an effective wavelength range between $812 \mathrm{~nm}$ and $833 \mathrm{~nm}$, which is less than half the specified bandwidth of the SLD source. The low modulation elsewhere in the spectrum is believed to be due to interference between front and back surfaces of the pellicle beam splitter (BS in Fig. 1) which leads to reduced spectral power of the reference and object beams for certain wavelengths. Figure 2(b) shows the magnitude of the Fourier transform of the signal in Fig. 2(a). The peak position is found between the integer frequency values produced by a discrete (fast) Fourier transform, by using an algorithm based on the continuous Fourier transform ${ }^{9}$. The continuous trace of the transform from which the peak is extracted is shown with a dashed line and the position of the peak is indicated with a vertical line.
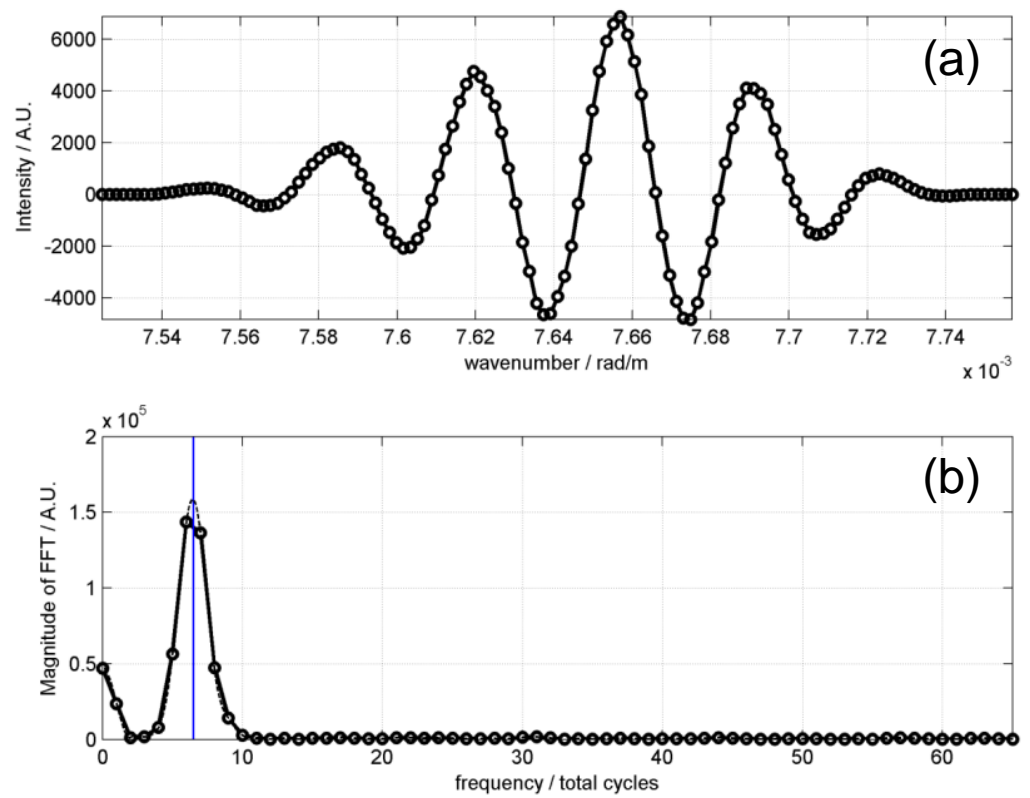

Figure 2. Spectral interference profile for one lenslet after removal of the dc component: a) intensity signal; b) Fourier Transform of (a). The output of the Fast Fourier Transform algorithm is shown as open circles, with the continuous Fourier transform overlaid as a dashed line, from which the peak position (vertical line) is evaluated. The peak frequency is then converted to optical path difference and height as described in points 9 and 10 in section 2.2.

\subsection{Translation of flat mirror}

Figure 3 shows the $35 \times 35$ point surface profiles obtained when a flat mirror was used as the object and shifted along the optical axis by a step of $100 \mu \mathrm{m} \pm 2 \mu \mathrm{m}$. 3675 independent coordinates were therefore measured. The mean displacement between the measured profiles was $97.62 \mu \mathrm{m}$. The field of view on the object corresponds to $3.5 \mathrm{~mm} \times 3.5 \mathrm{~mm}$ and the lateral resolution to $0.244 \mathrm{~mm}$. The spectral range $810-835 \mathrm{~nm}$ was effectively sampled with 131 pixels, i.e. $\delta \lambda=0.19$ $\mathrm{nm}$, and $\delta k=1.77 \times 10^{3} \mathrm{~m}^{-1}$, which corresponds to a depth range $h_{\mathrm{M}}=\pi / 2 \delta k=0.88 \mathrm{~mm}$. An rms value of $0.49 \mu \mathrm{m}$ was 
obtained for the height data after subtracting second order terms that arise due to lens distortions. In profilometry, the height measurement accuracy is usually expressed as the surface position uncertainty relative to the height measurement range, $\mathrm{rms} / h_{M}$, with $\mathrm{rms}$ the root mean square deviation of the measurement relative to the true figure. This ratio defines the inverse of the 'dynamic range'. In this system, they are equal to $5.3 \times 10^{-4}$ and 1871 , respectively.

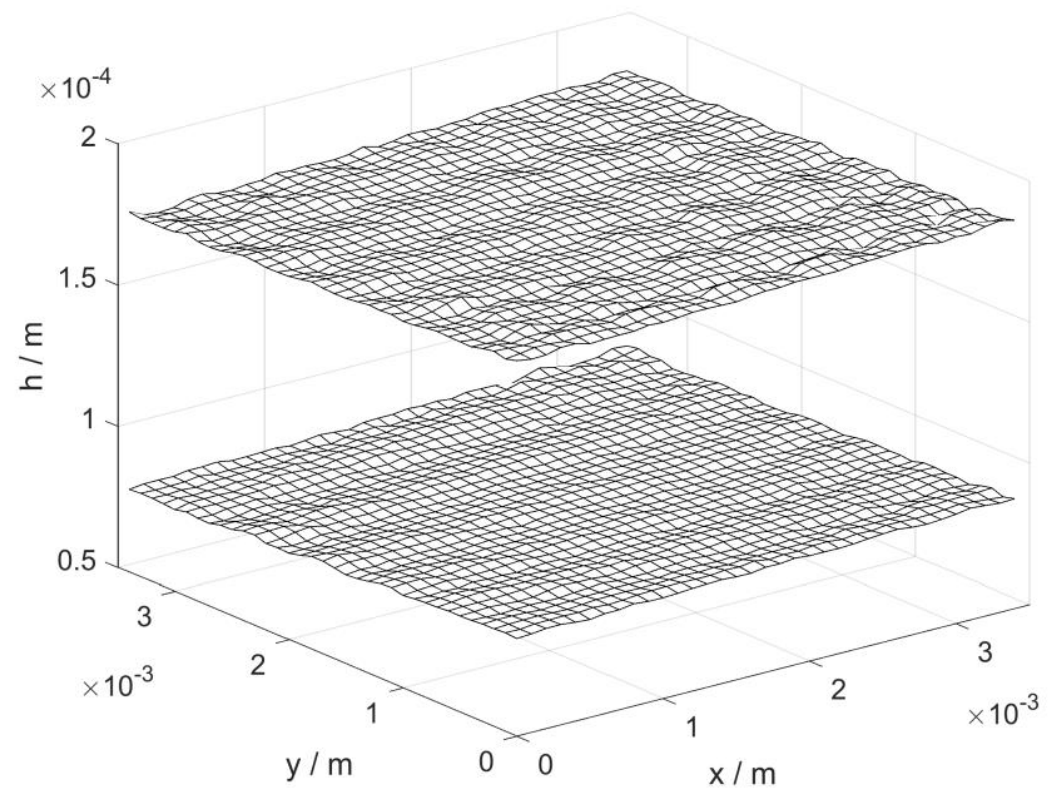

Figure 3. Surface height measurements of a flat mirror shifted $100 \pm 2 \mu \mathrm{m}$ normal to its surface by means of a micropositioning translation stage.

\subsection{Optically rough surface}

Many potential applications of the depth sensor involve surfaces that are rough on the scale of the wavelength of light. Such surfaces are more challenging due to speckle effects: dark speckles fall on some of the micro-lenses which lead to a very low signal to noise ratio of the spectra captured by the PDA and therefore no measurements at the corresponding object points. On the other hand, a greater range of surface tilts can be accommodated as compared to specularly reflecting surfaces because light is scattered over a wide range of angles. Figure 4(a) shows the height map of a surface grinding roughness standard (CLA1, from Rubert \& Company Ltd, Cheshire, UK), which has an average Ra roughness value of $3.2 \mu \mathrm{m}$. Figure 4(b) shows a cross section of Fig. 4(a) at row $n=15$. The spectral interferogram for one lenslet in the MLA, together with its Fourier transform are shown in Fig. 5. Compared to the signal from a smooth surface (Fig. 2), the intensity signal is less symmetrical and the Fourier peak is lower (notice the same vertical axis scale in Figs. 2(b) and 5(b)). The variation in modulation depth with wavenumber that causes the asymmetry can be attributed to the fact that speckle patterns decorrelate with changing wavelength, thus giving a non-constant object wave amplitude over the full bandwidth of the source ${ }^{10}$. Despite this, the measured profile of the sample (Fig. 4(a)) shows valid coordinate data over most of the field of view, with a residual height error with respect to the best fit plane of $13.87 \mu \mathrm{m}$. Only one point of low modulation due to a dark speckle was eliminated from the measured profile shown in Fig. 4(a). Spatial smoothing was not required for these results, unlike the case for the etalon based system from Ref. [7].

\section{CONCLUSIONS}

A high-throughput, single-shot areal profilometry system based on hyperspectral interferometry was presented. Spatial and spectral separation is achieved by means of a microlens array, a diffraction grating and a large area photodetector array. The optical configuration presented here uses significantly more of the pixels from a square or rectangular format 
image sensor than do previously reported systems ( $>40 \%$ instead of $\sim 2 \%$ ), with consequent benefits to the number of measured coordinates and/or the measurable depth range. The setup also avoids the need for the etalon used in previous systems and thus has significantly improved light efficiency. One limitation of the approach is that changes in surface slope have the effect of shifting the spectra on the photodetector array, eventually leading to cross talk between channels. An array of $35 \times 35$ independent points was measured simultaneously in a square grid with a pitch of $100 \mu \mathrm{m}$, covering a FOV of $3.5 \times 3.5 \mathrm{~mm} 2$ and with a depth range of $880 \mu \mathrm{m}$. Absolute height measurements were validated using a flat mirror mounted on a translation stage. This test achieved an rms height error of $0.49 \mu \mathrm{m}$, mainly dependent on lens aberrations which affect in particular the off-axis spectra. Measurement of a roughness gauge was also demonstrated.
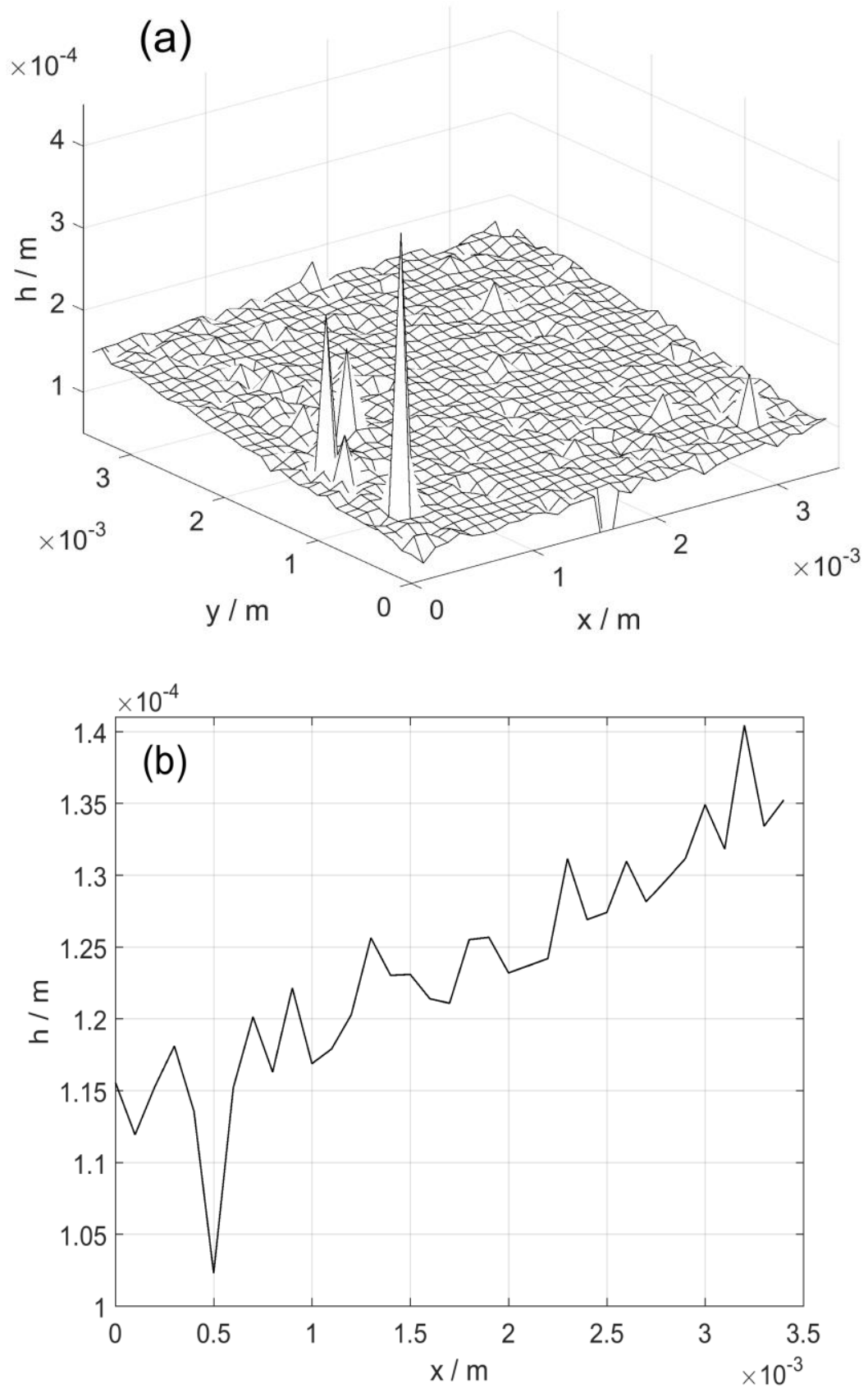

Figure 4. Surface profile of a surface roughness gauge: (a) measured surface profile from $35 \times 35$ lenslets of the MLA; (b) cross section of (a) along row $n=15$. 

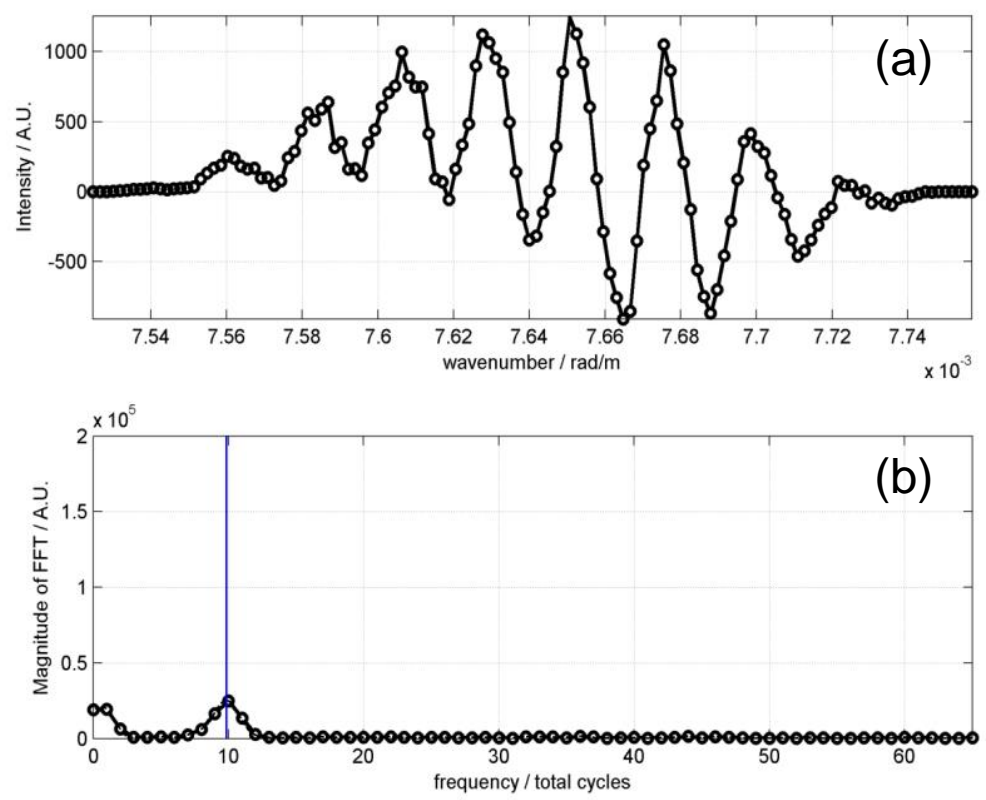

Figure 5. Typical intensity signal from one microlens (a) and its Fourier transform (b), for the case of a rough surface.

\section{ACKNOWLEDGMENTS}

P. D. Ruiz acknowledges The Royal Society (Industry Fellowship IF2012/R2) and industrial hosts Phase Vision Ltd. and Renishaw plc for their support. J. M. Huntley acknowledges the Engineering and Physical Sciences Research Council (EP/K018124/1) for its financial support.

\section{REFERENCES}

[1] Deck, L. and de Groot, P., "High-speed noncontact profiler based on scanning white-light interferometry," Appl. Opt., 33(31), 7334-7338 (1994).

[2] Sandoz, P. and Tribillon, G., "Profilometry by Zero-order Interference Fringe Identification," J. Mod. Opt., 40(9), 1691-1700 (1993).

[3] Dresel, T., Hausler, G. and Venzke, H., "3-dimensional sensing of rough surfaces by coherence radar,” Appl. Opt., 31, 919-925 (1992).

[4] Kuwamura, S. and Yamaguchi, I., "Wavelength scanning profilometry for real-time surface shape measurement," Appl. Opt., 36, 4473-4482 (1997).

[5] Takeda, M. and Yamamoto, H., "Fourier-Transform Speckle Profilometry - 3-Dimensional Shape Measurements of Diffuse Objects with Large Height Steps and/or Spatially Isolated Surfaces," Appl. Opt., 33(34), 7829-7837 (1994).

[6] Huntley, J.M., Widjanarko, T. and Ruiz, P.D., "Hyperspectral interferometry for single-shot absolute measurement of two-dimensional optical path distributions,” Meas. Sci. Tech. 21, 075304 (2010).

[7] Widjanarko, T., Huntley, J.M. and Ruiz, P.D., "Single-shot profilometry of rough surfaces using Hyperspectral Interferometry," Opt. Lett., 37(3), 350-352 (2012).

[8] Bacon, R. et. al., "3D spectrography at high spatial resolution. I. Concept and realization of the integral field spectrograph TIGER,” Astron. Astrophys. Suppl. Ser., 113, 347-357 (1995).

[9] Huntley, J.M., “An image processing system for the analysis of speckle photographs,” J. Phys. E 19(1), 43-48 (1986).

[10] Parry, G., [Laser Speckle and Related Phenomena], Springer Berlin, Heidelberg, 77-121 (1975). 